\title{
Empirical White Noise Processes and the Subjective Probabilistic Approaches
}

\author{
Péter Várlaki1,3, László Palkovicss ${ }^{2,3}$, András Rövid ${ }^{2 *}$ \\ ${ }^{1}$ Department of Control for Transportation and Vehicle Systems, Faculty of Transportation Engineering and Vehicle Engineering, \\ Budapest University of Technology and Economics, H-1111, Budapest, Stoczek street 2, Hungary \\ 2 Department of Automotive Technologies, Faculty of Transportation Engineering and Vehicle Engineering, Budapest University \\ of Technology and Economics, $\mathrm{H}-1111$ Budapest, Stoczek street 6, Hungary \\ ${ }^{3}$ Research Center of Vehicle Engineering, Széchenyi István University, H-9026 Győr, Egyetem tér 1., Hungary \\ * Corresponding author, e-mail: andras.rovid@gjt.bme.hu
}

Received: 15 September 2019, Accepted: 30 October 2019, Published online: 31 October 2019

\begin{abstract}
The paper discusses the identification of the empirical white noise processes generated by deterministic numerical algorithms.

The introduced fuzzy-random complementary approach can identify the inner hidden correlational patterns of the empirical white noise process if the process has a real hidden structure of this kind. We have shown how the characteristics of auto-correlated white noise processes change as the order of autocorrelation increases. Although in this paper we rely on random number generators to get approximate white noise processes, in our upcoming research we are planning to turn the focus on physical white noise processes in order to validate our hypothesis.
\end{abstract}

Keywords

empirical white noise processes, system identification, fuzzy-random view and possibility distributions, random number generators, $n$th order autocorrelation

\section{Introduction}

1.1 White noise process concerning the subjective probabilistic approaches ${ }^{1}$

The mathematical concept of white noise is well known from the probability theory (Wikipedia, 2019a). The essence is that the sequence of probability (random) variables that make up the temporal process is independent of each other at any given time. This is usually ensured by Kolmogorov's probability theory, where the probability calculus is based on the set theory (specifically the Sigma Algebras) (Kolmogorov, 1956). In alternative probability theories, independence of random variables can also be defined by interpreting the probability density functions of the two variables as the product of the probability density functions of the two variables. In this case, if we do not know them based on the value of zero of the correlation coefficient, the correlation can be interpreted as a kind of (non-Kolmogorovian) independence.

1 An extended version of the lecture at the University of Debrecen at the Conference on Time-series and their Application, 2019.
As is well known, alternative subjective probability schools often criticized Kolmogorov's theory because of the perceived compulsion of their sigma-based event systems (footnote on subjective probability). In addition to the different Bayesian approaches (Wikipedia, $2019 b)$ and the von Mises probability calculation method ${ }^{2}$ (von Mises, 1964), we may note the 'Treaties on Probability' (Keynes, 1921) written by J. M. Keynes, whose spirit contributed to the theory of "Expectations" of Keynes during the dynamic modeling of economic processes (thus exercising decisive influence on the history of the 20th century). John von Neumann's and Oscar Morgenstern's probability views on decision-making and game theory are to be included as well.

2 However, von Mises concerns the random time series of a Gambling system by pure mathematical approach as later Kolmogorov, no taking into consideration the practical empirical view, when they intend to prove the "impossibility of Gambling systems". Our approach, indirectly, intend to show a rather weak but existing possibility of a Gambling system. 
As for the white noise process in the case of Kolmogorov-based mathematical definition, the white noise process is a "blind", memoryless series of the random events lacking of correlation or "intelligence", not to mention any order or pattern, having an unpredictable future and unrecognizable past, as a series of chaotic events par excellence ${ }^{3}$.

In our paper, on the other hand, we intend to present a maybe possible "intelligent model" of the white noise processes primarily on the basis of the subjective approach of the "subjective probability", suggested by W. Pauli in describing the worldview of quantum physics. Thus, similarly to the subjective probabilistic approach, the undetached observer is organically linked to the observed events, and cannot be separated from them, because both the measurement system and the measurement processing model form a unit with the hypothetical patterns of the observed events. Before we go into this, it is worth mentioning this idea, presented by the famous Hungarian physicist Loránd Eötvös (Pauli, 1994) in his well-known lecture, preserved until today:

"Though the sight what nature provides (as Galiani tells) is just as if an infinite number of dice would make a pre-announced throw at any moment. I think, my Ladies and Gentlemen, the cubes of nature are leaded, and the greatest of magicians just smiles over our head! Dear congregation! One hundred years have passed since Galiani's apology ${ }^{4}$, a hundred years, that liberated mankind from the burdens of rough material work, let us draw by the rays of the sun and move fast with our distant brothers; and yet we are as stunned before Galiani's cubes as once Holbach's guests. One might call the lead by which the cube turns, coincidence, force or God, the inside of the cube remains a mystery to our minds." (The Yearbooks of the Hungarian Academy of Sciences, 1877-1882).

In connection with the alternative probabilistic approaches to the probability approach of physics, we mention the probabilistic interpretations of Karl Popper (Popper, 1985) and Lajos Jánossy. On the basis of the probability version of Lajos Jánossy's modern ether hypothesis, in the case of modeling the single-photon interferometer measurements, he advances the concept of the intelligent white noise model involuntarily (Ádám et al., 1955). Finally, we may also mention Bruno de Finetti's

3 See a cultural reflection of this "idea" in DeLillo (1985). 4 Nietzsche referred to him as "a most fastidious and refined intelligence" (Nietzsche and Middleton, 1996). subjective probability approach (De Finetti, 1974). In the 21 st century quantum extensions of de Finetti's representation theorem have been found to be useful in quantum information, in topics like quantum key distribution and entanglement detection (Caves et al., 2002).

From the point of view of quantum physics, Pauli interprets the idea of the "undetached observer" as opposed to Einstein's concept. He notes „Einstein so emphatically wishes to retain the ideal of the detached observer. To put it drastically the observer has according to this idea to disappear entirely in a discrete manner as hidden spectator, never as actor, nature being left alone in a predetermined course of events independent of the way in which the phenomena are observed. „Like the moon has a definite position." (Pauli, 1954)

Pauli writes in contrast ,the mathematical concept of probability arose from the Endeavour to render objective as far as possible the subjective expectation of a single event. To do this the expectation must be replaced by the objective average frequency of an event when it is repeated under like conditions." (Pauli, 1954)

Important is his conclusion that, in purely mathematical form Bernoulli's theorem is thus not as yet susceptible to empirical test, or this purpose it is necessary somewhere or other to include a rule for the attitude in practice of the human observer or particular the scientist, which takes account of the subjective factor as well, namely that the realization, even a single occasion, of a very unlikely event is rewarded from a certain point on as impossible in practice. At this point one finally reaches the limits which are set in principle to the possibility of carrying out the original program of the rational objectivation of the unique subjective expectation."

Pauli's note on the observation "... observation thereby takes the character irrational, unique actuality with unpredictable outcome. Moreover, the impossibility of subdividing the experimental arrangement without essentially altering the phenomenon, brings a new feature of wholeness into physical happenings. Contrasted with this irrational aspect of concrete phenomena which are determined in the actuality, there stands the rational aspect of an abstract ordering of the possibilities of statements by means of the mathematical concept of probability of the $\Psi$ function." (Pauli, 1954)

New Approach to Einstein: "The new theory on the contrary generalizes this classical ideals and postulates of the detached observer. Under the pressure of the physical facts summed up under the heading ,finiteness of the quantum 
of action", this logical generalization has emerged into a higher synthesis as a finally satisfactory solution of earlier contradictions. The mathematical inclusion, in quantum mechanics, of the possibilities of the natural event has turned out to be sufficiently whole framework to embrace the irrational actuality the singular event as well."

Finally, Pauli summarizes the spirit of the new approach in the following way. „The fact that the mathematical concept of probability has also justified itself in this new situation the noted by the term ,complementarity” seems to be highly significant. It appears that there corresponds to $i t$, at a very deep level, a reality in nature; it has provided a solid logical basis for the type of the natural law which generalizes the classical and deterministic explanation of the nature and provides the link between continuum (wave) and discontinuum (particle), and for which I have suggested the name "statistical correspondence" (Pauli, 1954).

Thus, the idea of complementarity appears in the objective and subjective probabilistic approach, and even more generally in the scientific knowledge. Albert SzentGyörgyi writes about this: “... a discovery must be, by definition, at variance with existing knowledge", divided scientists into two categories: the Apollonians and the Dionysians. He called scientific dissenters, who explored "the fringes of knowledge", Dionysians. He wrote, "In science the Apollonian tends to develop established lines to perfection, while the Dionysian rather relies on intuition and is more likely to open new, unexpected alleys for research...The future of mankind depends on the progress of science, and the progress of science depends on the support it can find. Support mostly takes the form of grants, and the present methods of distributing grants unduly favor the Apollonian". (Szent-Györgyi, 1972)

It is interesting to note that Albert Szent-Györgyi follows Nietzsche's idea of 'Appollonian-Dionysian' or certainly its Jungian reinterpretation, as the latter one was attempted to apply for the identification of the psychological types by C. G. Jung (1976).

Interestingly, the intelligent white noise model itself has the above-mentioned approach to complementarity, perhaps first in the history of thinking in the con-

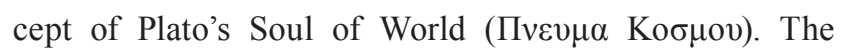
idea of the same and different, indivisible and divisible of this Platonic Complementarity can be perceived in the Apollonian and Dionysian idea of the mythology or its version of the Delphi religion. Obviously, this idea reflects on the revitalization of Nitzsche's experiment.
The harmony and simplicity of the whole picture of the intelligent concept of white noise suggests in a complementary way the eternal Apollonian spirit, while the realization of the process, leading to the components or the bands (measured data series) suits the Dionysian "half" (concerning the approach) of complementarity in an allegoric or symbolic form.

The subjective probability approach can also be compared to the theory of fuzzy sets and their membership functions, which can be interpreted in a complementary way, since the membership function showing the possibility distribution normally provides a probability density function not necessarily in a Kolmogorov sense. In this way, it gives the possibility to the interpretation of a sort of probability feature of the concept of the intersection of central importance in the fuzzy logic theory.

However, Kolmogorov's random and Zadeh's fuzzy (or fuzzy-random) concept of independence is clearly distinguished. In this case, if the combined probability density functions are derived from the multiplication of the density function of the two variables, the correlation coefficients interpreted on the intersections already show a value different from zero in the case where the variance load or probability density functions are not the same. Thus, the complete independence in the sense of Kolmogorov is only valid if the inclusion or probability density functions of the two possibilistic or probabilistic variables are identical for the not zero Alpha-cuts of the original membership function.

It could mean a (new) complementary representation theory for the uncertainty approach which can be simultaneously treated as possibilistic as probabilistic one (Baranyi and Várlaki, 2015; Bhargava (2013). Consequently, the characteristic membership function can be concerned according to this complementary principle both probabilistic and possibilistic distributions. Namely, if a possibilistic distribution (function) is represented by a fuzzy membership function, then its normalized version can represent a probability distribution (density function) in a traditional sense of the concept. Therefore, the possibilistic and probabilistic approaches (similarly to the wave particle complementary principle) are jointly valid representation of a general uncertainty which are undivided, inseparable of each other. This could mean a complementary uncertainty representative theory, where the uncertainty representative function is both complementary-like possibilistic as well as probabilistic distribution. If the possibilistic distribution is represented by a fuzzy membership function then at the same time its normalized version represents a probability density 
function, as well in a traditional way (Fullér et al., 2011; 2012). Thus, the possibilistic and probabilistic approaches (similarly to the wave-particle complementarity approach) show up simultaneously, jointly and inseparably from each other (Papp et al., 1998). This could represent the basis of a new joint probabilistic-possibilistic theory.

\section{The correlation analysis in case of empirical white noise processes}

The above discussed approach can be applied for the definition or the interpretation of complementary fuzzy-random (or possibilistic-probabilistic) processes, as well. In Figs. 1, 2 it can be seen, that besides arbitrary generation (division or partition) the system of the band intervals can be generated by the concept of the alpha-cut (of a suitable fuzzy membership function) induced intervals. In this case the white noise process realization can be characterized (represented) naturally complementarily by both possibilistic (fuzzy) and probabilistic (random) processes. Consequently, the principle of the above complementarity is unconditionally necessary for the realistic modeling of the data for the experimental white noise processes. Now let us concern the new white noise "idea" in the case of the concept of independence with the above discussed "inseparable valid" complementary fuzzy-random approach. This concept of independence is the basis of the new type of deconstructive modeling of the empirical white noise processes which were treated as traditional random phenomena. Following this approach we can define and interpret new kinds of fuzzy-random processes for the representations of a relative wide class of stochastic processes in the traditional sense of the word. In this case the stochastic processes can be generated alternatively from so called source white noise processes as we can see this kind of representation in the description of the ARMA processes.

In the practice the entire image of the empirical autocorrelation function has been considered in the calculations of the autocorrelation. Let $u(t)$ be the realization of the empirical white noise process (observed series of numbers) (Michaletzky et al., 1998).

$R_{u}(\tau)=\frac{1}{T} \sum_{t=1}^{T}(u(t)-\bar{u})(u(t-\tau)-\bar{u})$

where $\bar{u}=\frac{1}{T} \sum_{t=1}^{T} u(t)$.

The autocorrelation function of the "fragmented" realization $u_{n}(t)$ considered for time period $T$ is as follows:
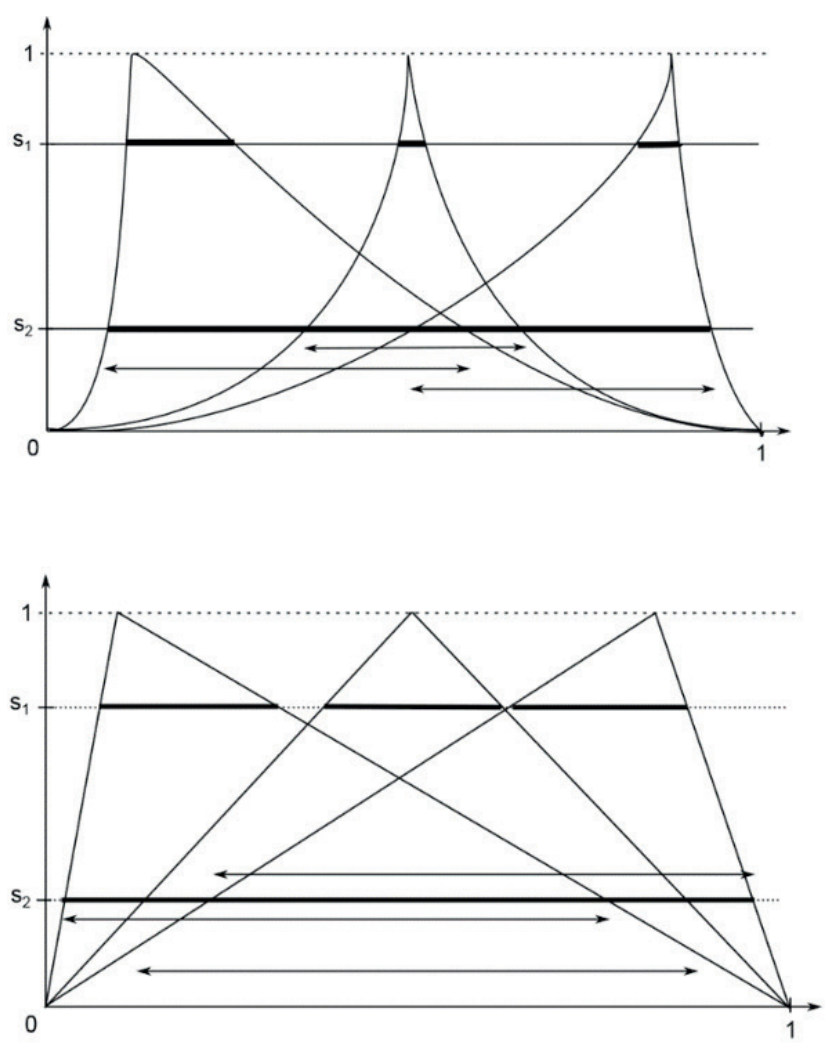

Fig. 1 An important aspect of the probabilistic approach is that in case of fuzzy random white noise processes (considering given, concrete realizations) the distributions and their density functions - interpreted in a fuzzy-random way - may change or might be different.

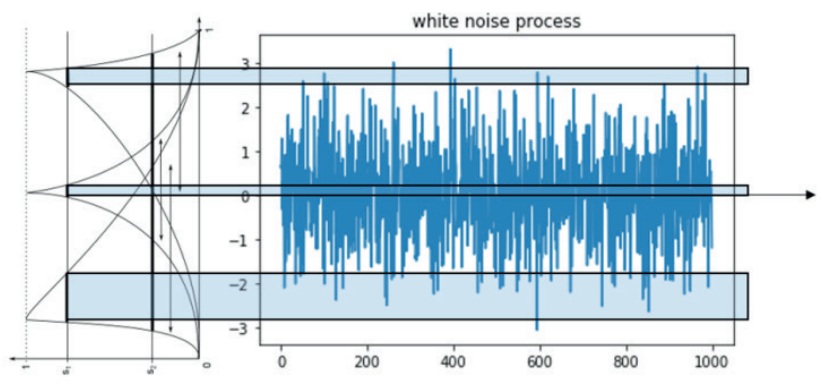

Fig. 2 The illustration of the above idea for a specific realization.

$R_{n, n}(\tau)=\frac{1}{T} \sum_{t=1}^{T}\left(u_{n}(t)-\bar{u}_{n}\right)\left(u_{n}(t-\tau)-\bar{u}_{n}\right)$

where $\bar{u}_{n}=\frac{1}{T} \sum_{t=1}^{T} u_{n}(t)$.

The fragmented $u_{n}(t)$ process might be given in the following form, as well:

$u_{n}(t)=u(t) I\left(u(t) \in \Delta_{n}\right)$

where the indicator function

$I\left(u(t) \in \Delta_{n}\right)=\left\langle\begin{array}{ll}1 & u(t) \in \Delta_{n} \\ 0 & \text { otherwise }\end{array}\right.$. 
Here $\left\{\Delta_{n}\right\}$ denotes the arbitrarily clustered system of bands.

Under the cross-correlation function of two arbitrary bands the following relation is meant:

$$
R_{n, m}(\tau)=\frac{1}{T} \sum_{t=1}^{T}\left(\left(u_{n}(t)-\bar{u}_{n}\right)\left(u_{m}(t-\tau)-\bar{u}_{m}\right)\right)
$$

where $\bar{u}_{m}=\frac{1}{T} \sum_{t=1}^{T} u_{m}(t)$.

Here the normalized auto- and cross-correlation functions

$$
r_{n, n}(\tau)=\frac{R_{n, n}(\tau)}{\sigma_{n}^{2}}
$$

where $\sigma_{n}^{2}=\frac{1}{T} \sum_{t=1}^{T}\left(u_{n}(t)-\bar{u}_{n}\right)^{2}$.

Furthermore,

$$
r_{n, m}(\tau)=\frac{R_{n, m}(\tau)}{\sigma_{n} \sigma_{m}}
$$

where

$$
\begin{aligned}
& \sigma_{m}^{2}=\frac{1}{T} \sum_{t=1}^{T}\left(u_{m}(t)-\bar{u}_{m}\right)^{2} \\
& 0 \leq r_{n, n} \leq 1 \wedge 0 \leq r_{n, m} \leq 1 .
\end{aligned}
$$

In the followings, let us consider the normalized autoand cross-correlation functions only when dealing with calculations or providing illustrations. Since the autocorrelation and cross-correlation functions of empirical white noise process realizations form stationary random series, the autocorrelation functions derived through automorphic-like transformations (autocorrelation operators) might be considered to be stationary random series, as well (for a reduced time period).

In this case let

$$
z_{n}(\tau)=R_{n, n}(\tau), \bar{z}_{n}(\tau)=\frac{1}{T^{\prime}} \sum_{\tau=1}^{T^{\prime}} z_{n}(\tau) .
$$

The first order automorphic autocorrelation function is as follows:

$$
w_{z_{n}}\left(\tau^{\prime}\right)=R_{z_{n}}\left(\tau^{\prime}\right)=\frac{1}{T^{\prime}} \sum_{\tau=1}^{T^{\prime}}\left(z_{n}(\tau)-\bar{z}_{n}\right)\left(z_{n}\left(\tau-\tau^{\prime}\right)-\bar{z}_{n}\right) .
$$

In this case the normalized first order automorphic autocorrelation function is as follows:

$$
r_{z_{n}}(\tau)=\frac{R_{z_{n}}(\tau)}{\sigma_{z_{n}}^{2}}, 0 \leq r_{z_{n}} \leq 1
$$

The second order automorphic autocorrelation function might be expressed as:

$$
w_{z_{n}}^{\prime}\left(\tau^{\prime \prime}\right)=R_{z_{n}}\left(\tau^{\prime \prime}\right)=\frac{1}{T^{\prime \prime}} \sum_{\tau^{\prime}=1}^{T^{\prime \prime}}\left(w_{z_{n}}\left(\tau^{\prime}\right)-\bar{w}_{z_{n}}\right)\left(w_{z_{n}}\left(\tau^{\prime}-\tau^{\prime \prime}\right)-\bar{w}_{z_{n}}\right)
$$

where $\bar{w}_{z_{n}}=\frac{1}{T^{\prime \prime}} \sum_{\tau^{\prime}=1}^{T^{\prime \prime}} w_{z_{n}}\left(\tau^{\prime}\right)$ and the standard deviation

$\sigma_{w_{z_{n}}}^{2}=\frac{1}{T^{\prime \prime}} \sum_{\tau^{\prime}=1}^{T^{\prime \prime}}\left(w_{z_{n}}\left(\tau^{\prime}\right)-\bar{w}_{z_{n}}\right)^{2}$

In this case the second order automorphic autocorrelation function might be expressed as:

$r_{w_{z_{n}}}\left(\tau^{\prime \prime}\right)=\frac{R_{w_{z_{n}}}\left(\tau^{\prime \prime}\right)}{\sigma_{w_{z_{n}}}^{2}}, 0 \leq r_{w_{z_{n}}} \leq 1$

Since such obtained cross-correlation functions show stationary or nearly stationary realizations (without considering the central point), thus even for these derived realizations the autocorrelation functions might be calculated, as well.

$$
z_{n, m}(\tau)=R_{n, m}(\tau)
$$

In this case the first order automorphic autocorrelation function of the cross-correlation function of two bands is the following:

$$
R_{n, m}(\tau)=\frac{1}{T^{*}} \sum_{t=1}^{T^{*}}\left(z_{n, m}(t)-\bar{z}_{n, m}\right)\left(z_{n, m}(t-\tau)-\bar{z}_{n, m}\right)
$$

where

$$
\begin{aligned}
& \bar{z}_{n, m}(t)=\bar{z}_{n, m}=\frac{1}{T^{*}} \sum_{t=1}^{T^{*}} z_{n, m}(t) \\
& \sigma_{z_{n, m}}^{2}=\frac{1}{T^{*}} \sum_{t=1}^{T^{*}}\left(z_{n, m}(t)-\bar{z}_{n, m}\right)^{2} .
\end{aligned}
$$

In this case the first order automorphic autocorrelation function of the cross-correlation function might be written as:

$$
r_{n, m}(\tau)=\frac{R_{n, m}(\tau)}{\sigma_{z_{n, m}}^{2}}, 0 \leq r_{n, m}(\tau) \leq 1
$$

By continuing the automorphic mapping process, the second order autocorrelation function might be considered as cross-correlation function if

$$
w_{n, m}\left(\tau^{\prime}\right)=R_{n, m}\left(\tau^{\prime}\right)
$$

then $R_{w_{n m, n m}}\left(\tau^{\prime}\right)$ and for the normalized case $r_{w_{n, m}}\left(\tau^{\prime}\right)$ where $R$ stands for the autocorrelation operator considered 
for appropriate processes. Let us finally consider the randomly derived cross-correlation function of two cross-correlation functions:

$$
w_{z_{m n} z_{j j}}^{\prime}\left(\tau^{\prime}\right)=R_{z_{m m} z_{j j}}=\frac{1}{T} \sum_{t=1}^{T}\left(w_{z_{n m}}(t)-\bar{w}_{z_{n n}}\right)\left(w_{z_{l j}}\left(t-\tau^{\prime}\right)-\bar{w}_{z_{k j}}\right) .
$$

In this case we may take the generative like autocorrelation function of the above nearly stationary realization:

$$
\begin{aligned}
w_{z_{m n} z_{k j}}^{\prime}\left(\tau^{\prime \prime}\right) & =R_{z_{m m} z_{j j}} \\
& =\frac{1}{T} \sum_{t=1}^{T}\left(w_{z_{m n} z_{k j}}(t)-\bar{w}_{z_{m n} z_{j j}}\right)\left(w_{z_{n m} z_{j j}}\left(t-\tau^{\prime \prime}\right)-\bar{w}_{z_{m n} z_{k j}}\right)
\end{aligned}
$$

where $\bar{w}_{z_{m n} z_{k j}}=\frac{1}{T} \sum_{t=1}^{T} w_{z_{m m} z_{k j}}(t)$

Let us denote the generative autocorrelation functions obtained by second order automorphic mapping as $R_{z_{m m} z_{k j}}$ and in normalized case as $r_{z_{m m} z_{j}}$.

As we can see from the following results the introduced automorphic auto-correlational maps (where the autocorrelation operator

$$
R_{z u}(\tau)=E\left[\left(z_{t}-E(z)\right)\left(u_{t-\tau}-E(u)\right)\right],
$$

where $E$ stands for the symbol to get the expected value) are suitable for the modeling of the hidden generative processes controlling the periodical realization of the empirical white noise process. This hypothetical observation is very similar to the Pauli's idea of automorphism in the case of the quantum physical descriptions.

„It is the automorphism for the ascribing to others one's own characteristics, an isomorphism of an algebraic system with itself, in other words for a process in which the inner symmetry, the wealth of (hidden) relations of a system reveals itself." (Meier, 2002)

Between the obtained generative processes of first and second order we can calculate cross-correlation functions again in form of stationary random data processes. These processes or autocorrelation phenomena can be considered as complex double cross-correlation generative processes. Concerning the bands we can create huge amount of generative processes, which in case of a hypothetic continuous concept, naturally, mean infinitely many generative processes. Then the question rises, until when can generative processes be created. On the basis of our experiments using the stationarity hypothesis of generative processes the measure of stationarity is slowly vanishing.
The measure of stationarity might be measured by the ratio of the area falling below the boundary curve of the signal (considered for a finite time interval) and the rectangular area enclosing the fully stationary, normalized realizations in the same time interval.

\section{Simulation experiments}

\subsection{The applied numerical approaches}

The calculation of the autocorrelation and cross-correlation can be performed in the time domain or in the frequency domain. In the latter case we rely on the convolution theorem when calculating the convolution of random processes. The spectrum of the realization was determined by Fast Fourier Transformation (FFT). The overlap between signals as well as their lengths were also taken into account during calculations. We have obtained identical numerical data both in frequency and time domain.

\subsection{Results and their interpretation}

Among the white noise processes obtained by random number generators let us first consider the one generated by the "randn" function in Matlab available to generate normally distributed random numbers. (It's important to realize that "random" numbers in MATLAB are not unpredictable at all, but are generated by a deterministic algorithm. The algorithm is designed to be sufficiently complicated so that its output appears to be an independent random sequence to someone who does not know the algorithm, and can pass various statistical tests of randomness (Mathworks, 2019). By our approach the deterministic properties of random processes generated by deterministic algorithms might be identified (Savage, 1954).

In Fig. 3 the mirrored bands can be followed falling inside the amplitude range of the signal, where the white noise 'sub-processes' composed from so called "broken"

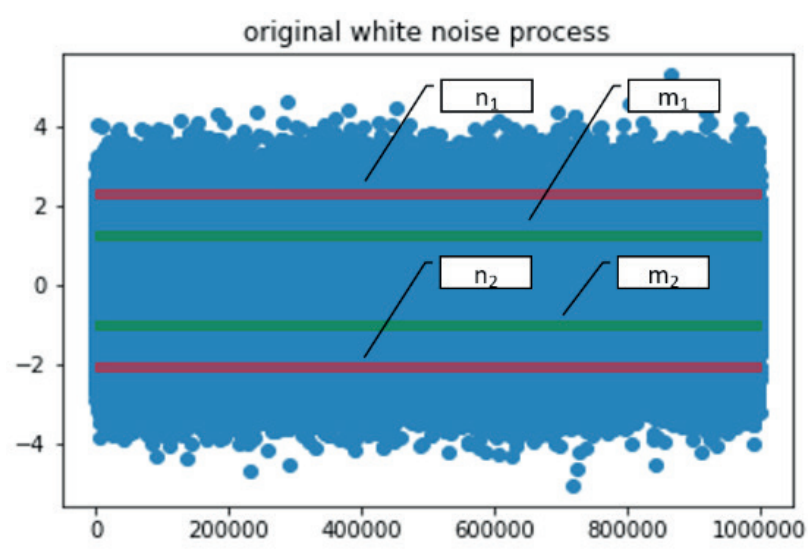

Fig. 3 The original white noise process 
samples inside these bands are investigated. In Fig. 4 the autocorrelation function of the original white noise is illustrated. Although the autocorrelation function shows a white noise like realization, it can be considered as a good approximation (due to the small fluctuations, small standard deviation) of the Dirac delta function as the normalized autocorrelation function of the continuous white noise process.

By forming the autocorrelation function of such a realization a new generative like autocorrelation function is yielded with a transformed time-shift.

As we can expect of the centre it may be considered as a new realization of a ,background generative" stationary process in a reduced time domain. The autocorrelation function of this new realization can be calculated again.

Let us consider the cross-correlation function of the first order generative process illustrated in Fig. 5, 6 with the realization of the first order generative process observable in the mirrored bands (see Figs. 7-9). Such kind of obtained cross-correlation function may also be considered as a realization of a stationary (derived white noise) process in the already transformed time domain interval. The same

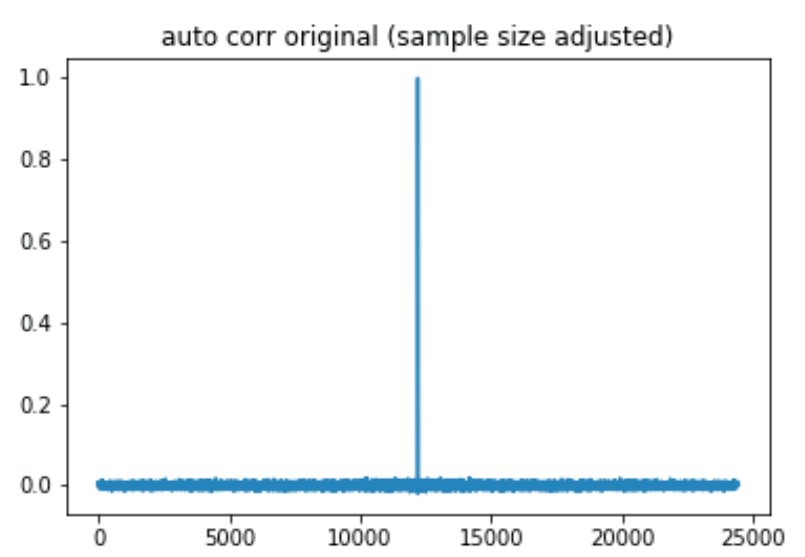

Fig. 4 The autocorrelation function of the original white noise process

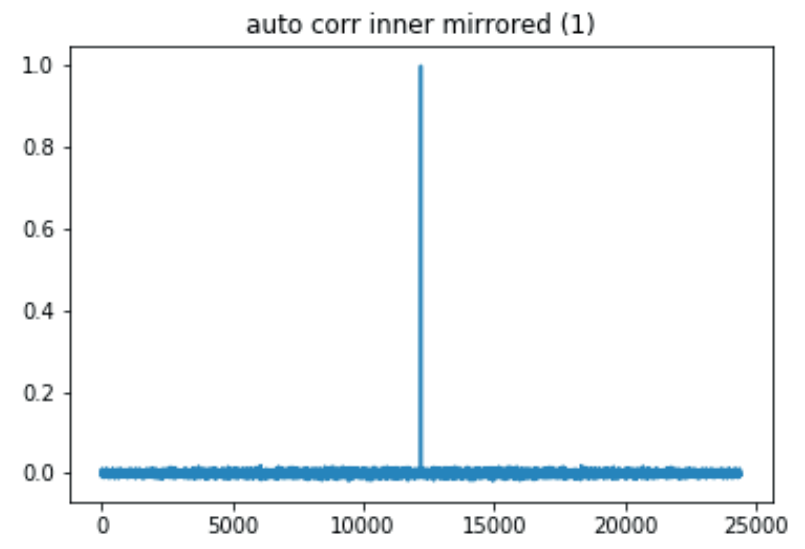

Fig. 5 The autocorrelation function of the band $m_{1}$ transformational autocorrelation mappings (realizations) can be observed in case of time series falling inside the outer mirrored (relatively narrow) bands (see Figs. 10-16). The two outer mirrored autocorrelation functions (as stationary realizations) reflect new (second and third order) generative processes (with new autocorrelation mappings).

It can be seen that the second order autocorrelation function of the inner outer cross-correlations (double cross-correlations) does not yield the image of a stationary realization anymore, but a real function-like autocorrelation function (reflecting the periodicity of the generation rule).

Let us consider now the fuzzy measure of stationarity. Our definition is as follows: The fuzzy measure of stationarity is the ratio (for a finite time value) of the area determined by the minimal boundary curve and the minimal bounding rectangle. This definition is based on the hypothetical constant value of the mean and variance of the white noise realization for the mentioned finite Time value. See the illustration in Fig. 17 and 18. Thus, to all generative autocorrelation functions one may assign a fuzzy measure of stationarity falling between 0 and 1 .

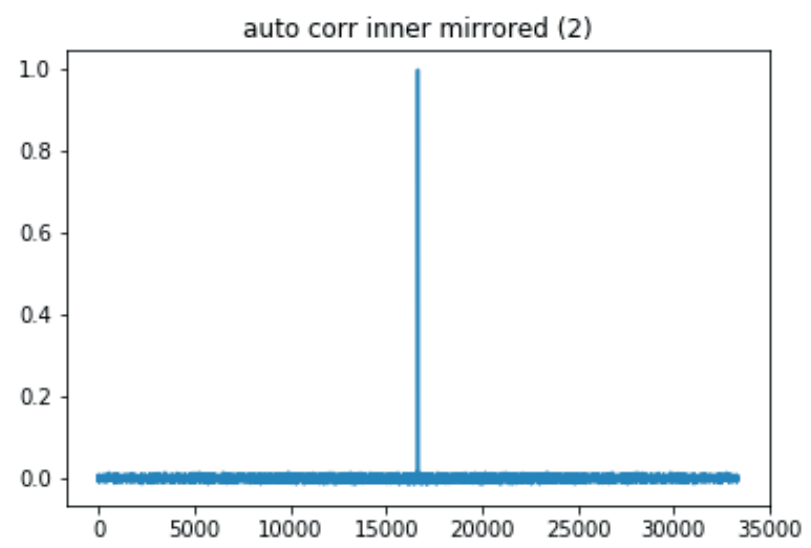

Fig. 6 The autocorrelation function of the band $\mathrm{m}_{2}$

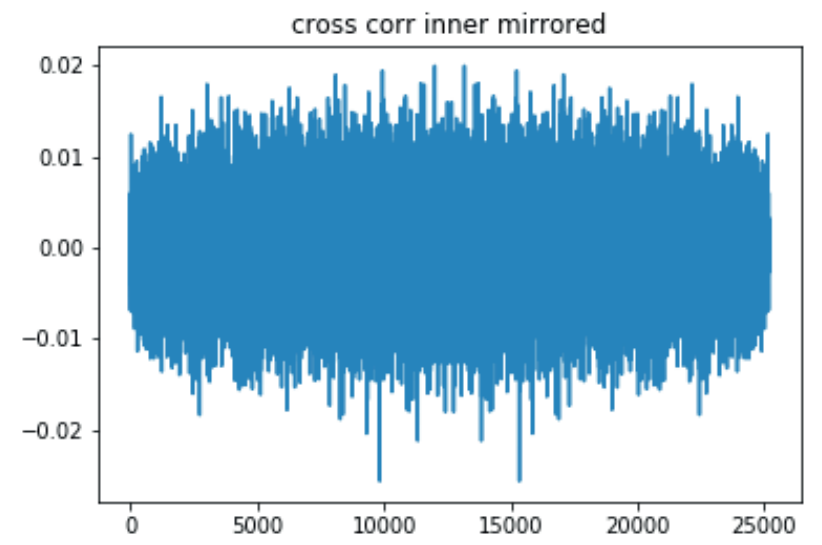

Fig. 7 The cross-correlation of bands $m_{1}$ and $m_{2}$ 


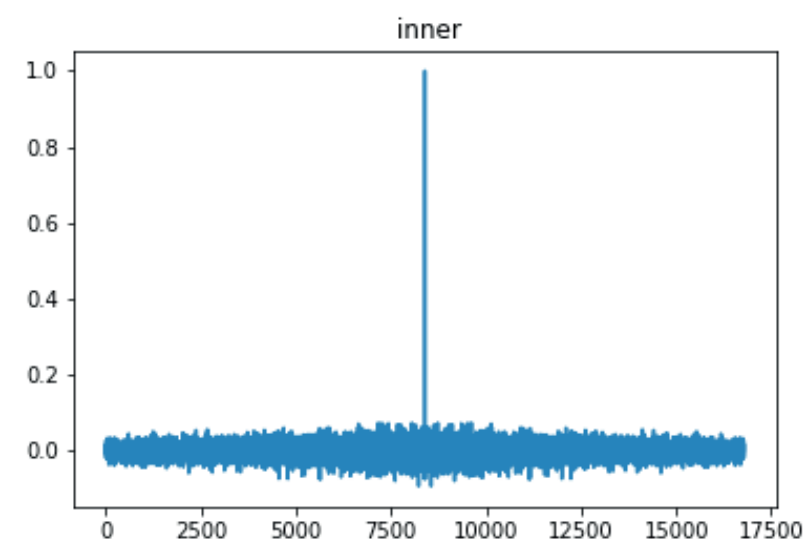

Fig. 8 First order autocorrelation function of cross-correlation of bands $\mathrm{m}_{1}$ and $\mathrm{m}_{2}$

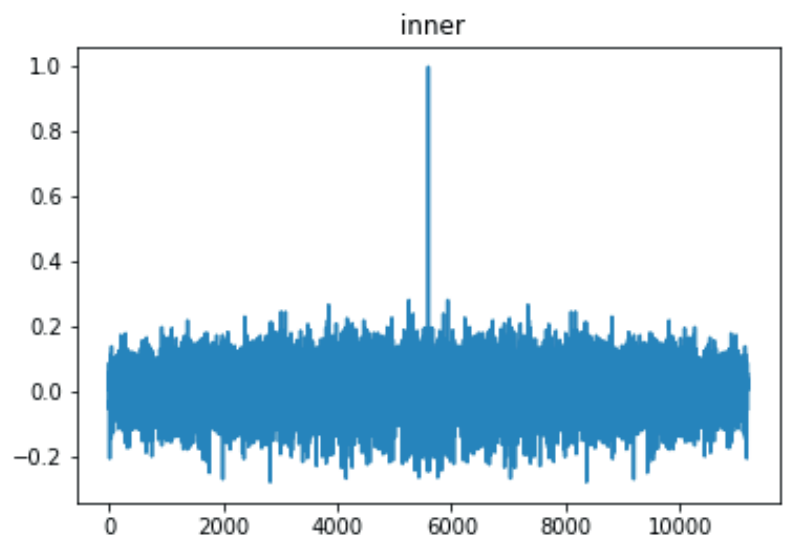

Fig. 9 Second order autocorrelation function of cross-correlation of bands $\mathrm{m}_{1}$ and $\mathrm{m}_{2}$

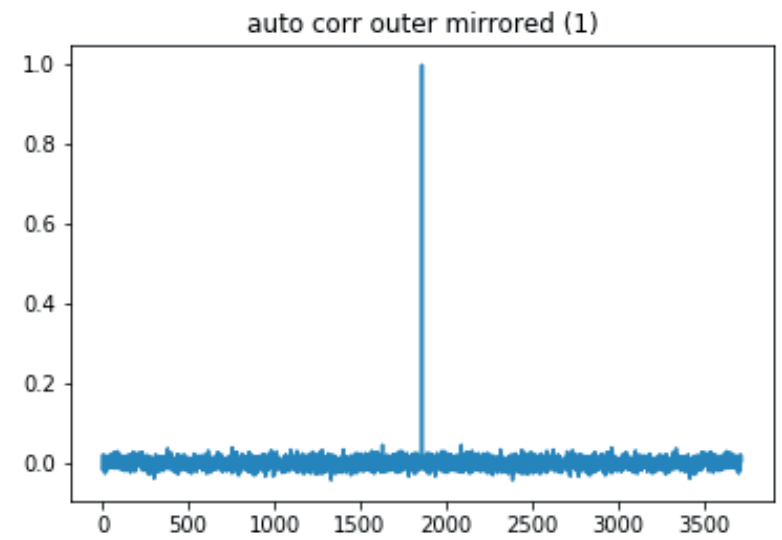

Fig. 10 The autocorrelation function of the band $n_{1}$

\subsection{MWC1 random number generator}

The same can be seen in Figs. 19-27 in case of another type of random number generator, the so called MultiplyWith-Carry generator (MWC) which was proposed by George Marsaglia in 1994 and analyzed by Couture and

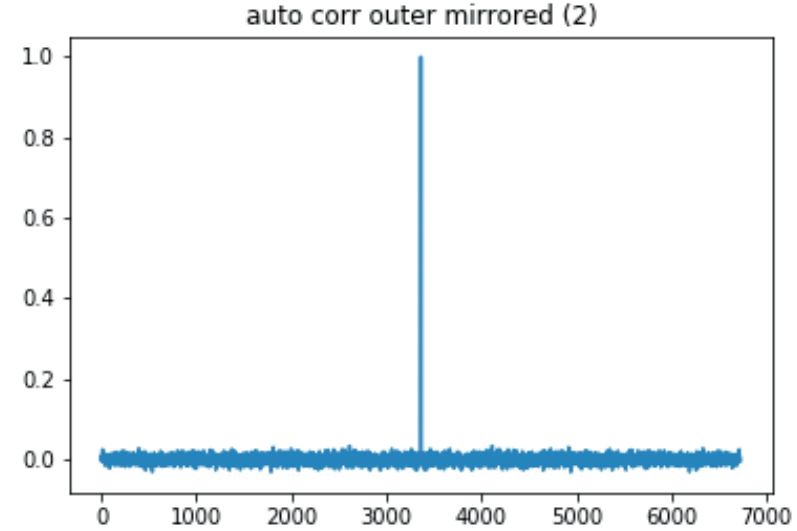

Fig. 11 The autocorrelation function of the band $n_{2}$

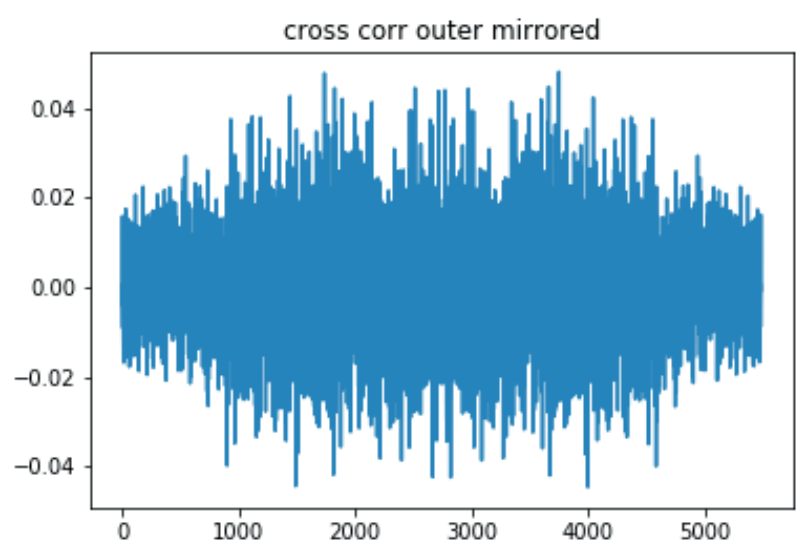

Fig. 12 The cross-correlation of bands $n_{1}$ and $n$

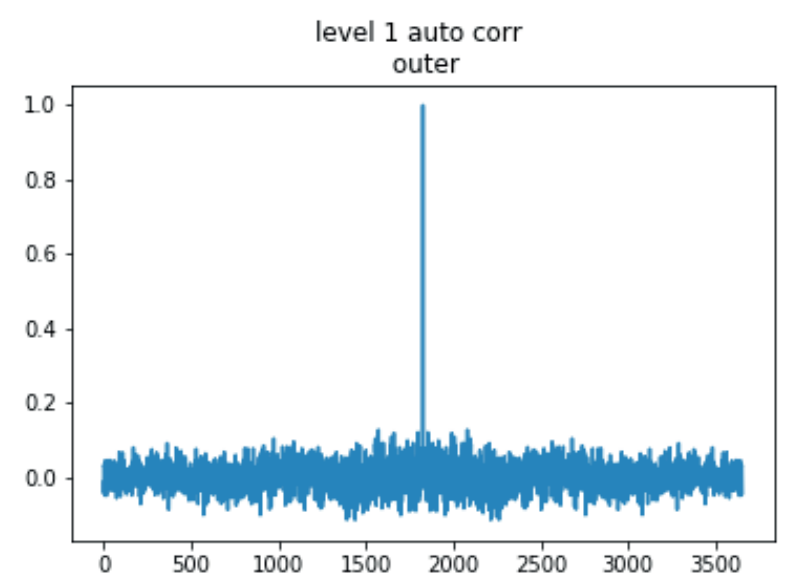

Fig. 13 First order autocorrelation function of cross-correlation of bands $n_{1}$ and $n$

L'Ecuyer in 1997. MWC was proposed as a modification of the Add-With-Carry (AWC) generator (CREłU, 2012).

In addition to calculating the cross-correlation of autocorrelation functions as realizations (obtained from narrow band realizations) we can also perform new 


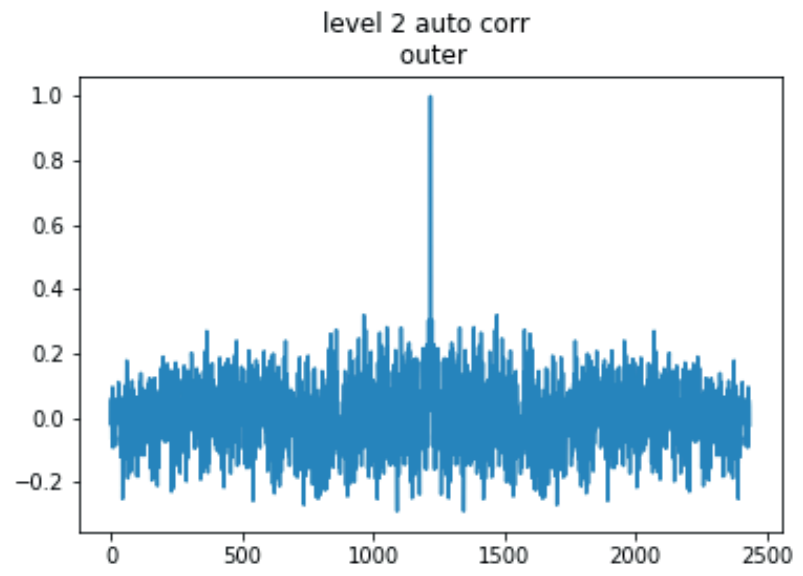

Fig. 14 Second order autocorrelation function of cross-correlation of bands $\mathrm{n}_{1}$ and $\mathrm{n}_{2}$

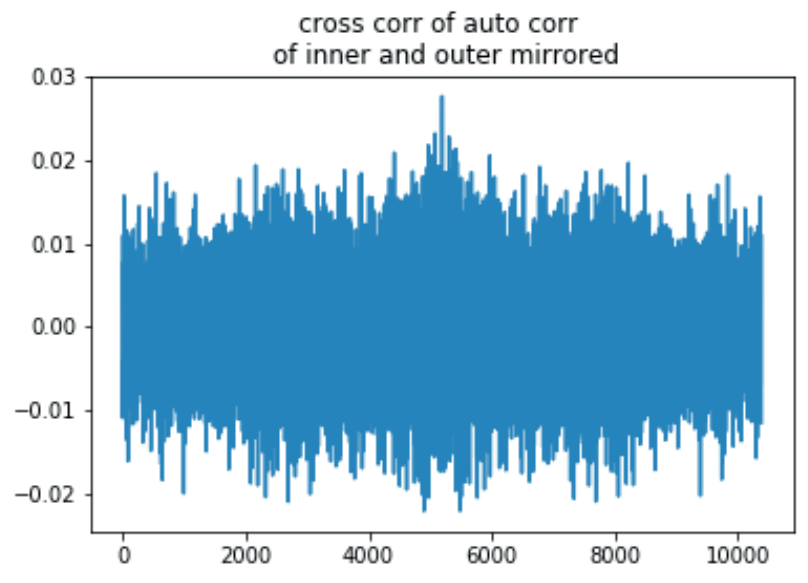

Fig. 15 The cross-correlation of second order autocorrelation of inner and outer bands

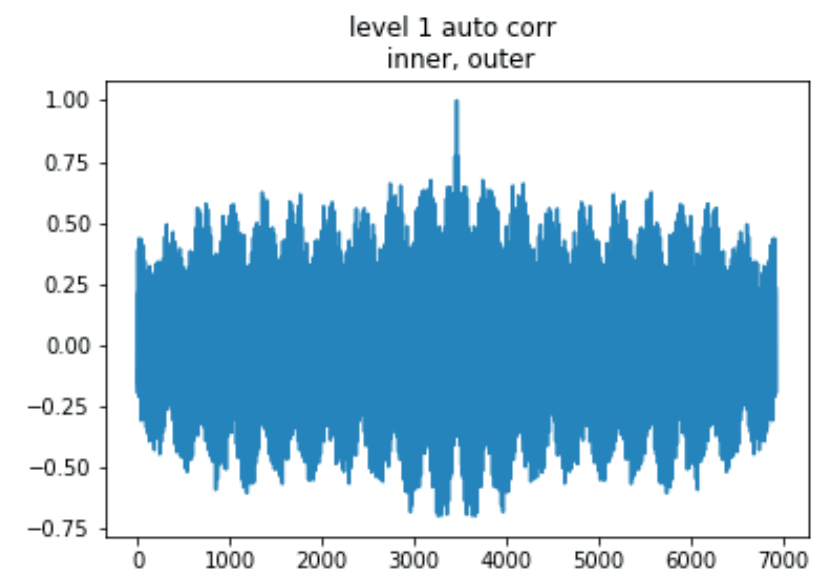

Fig. 16 First order autocorrelation of function illustrated in Fig. 15.

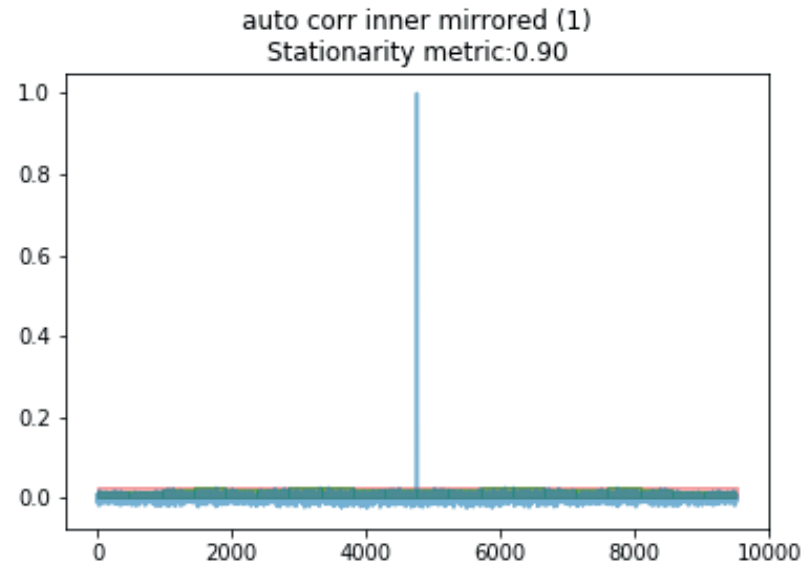

Fig. 17 Illustration of the first order autocorrelation of the 1st inner mirror band with the indicated enclosing regions (bounding rectangle and the region defined by the approximated boundary curve) used for stationarity metric estimation

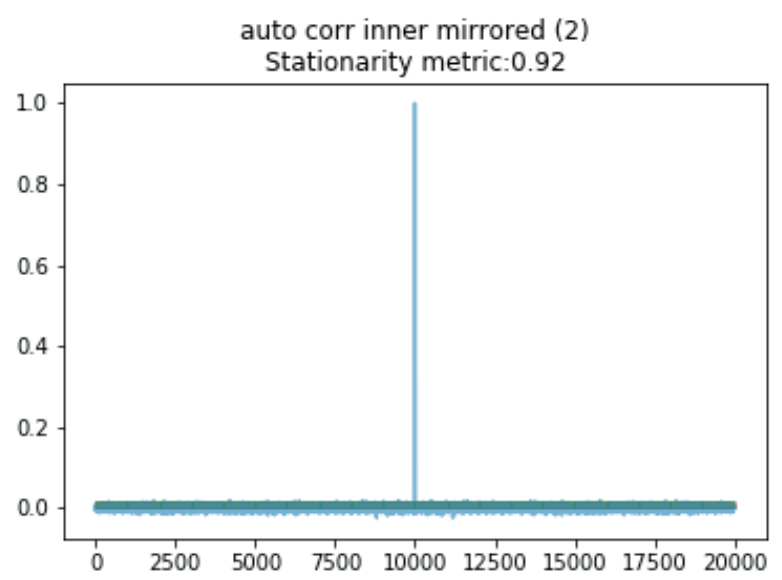

Fig. 18 Illustration of the first order autocorrelation of the 2nd inner mirror band with the indicated enclosing regions (bounding rectangle and the region defined by the approximated boundary curve) used for stationarity metric estimation

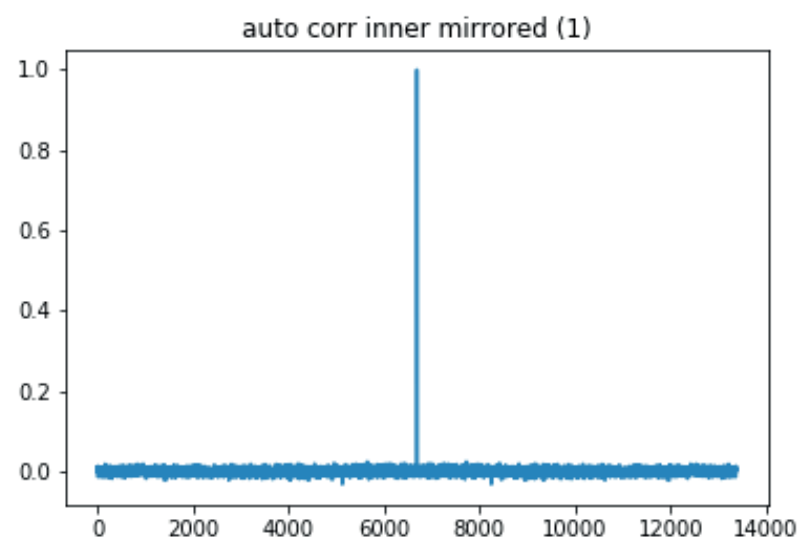

Fig. 19 The autocorrelation function of the band $m_{1}$ 


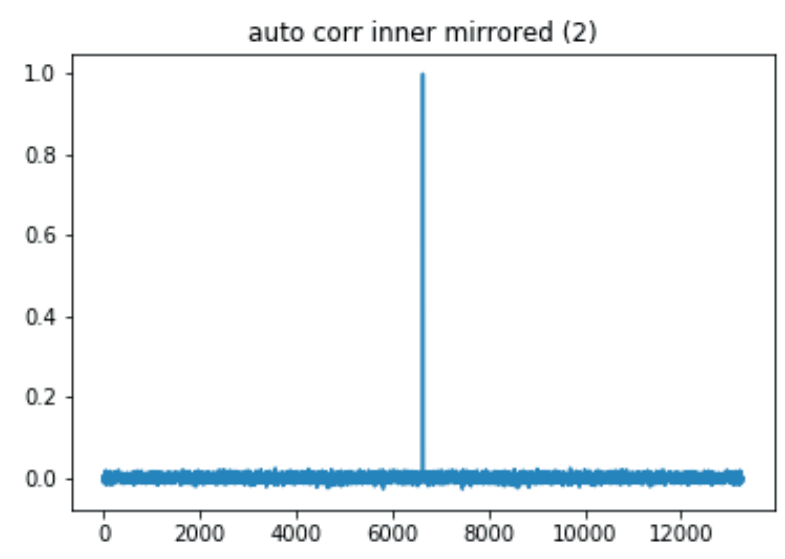

Fig. 20 The autocorrelation function of the band $\mathrm{m}_{2}$

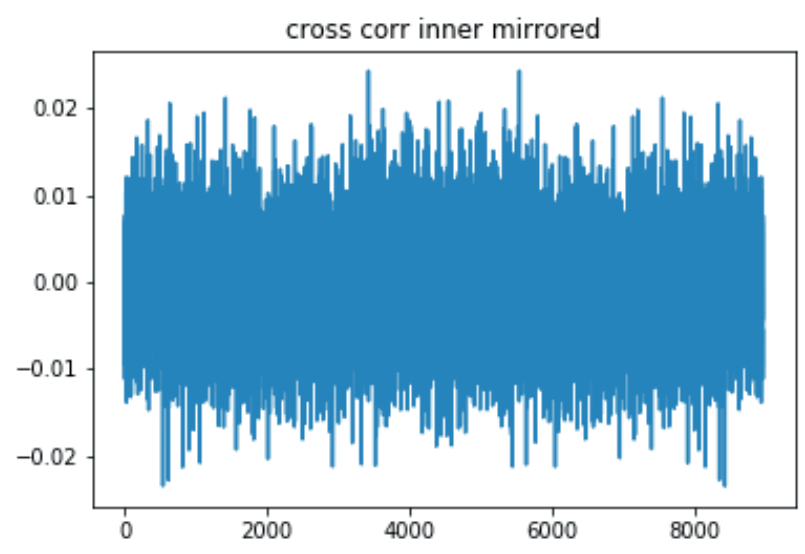

Fig. 21 The cross-correlation of bands $m_{1}$ and $m_{2}$

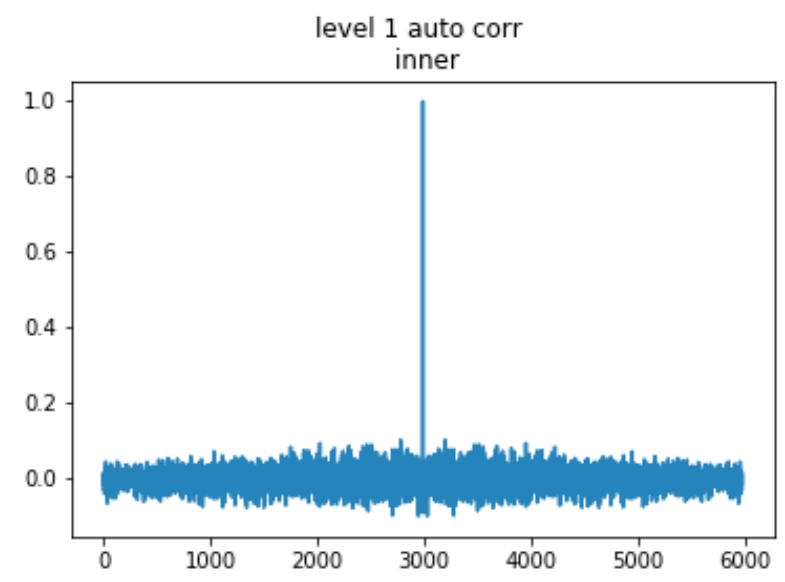

Fig. 22 First order autocorrelation function of cross-correlation of bands $\mathrm{m}_{1}$ and $\mathrm{m}_{2}$

autocorrelation mappings as unique automorphism on them until the stationarity characteristic holds with a good approximation. The original white noise process is not visualized here, since compared to previous examples the differences cannot be clearly recognized.

From the above experimental results and their illustrations we can conclude hypothetically that the homogenous

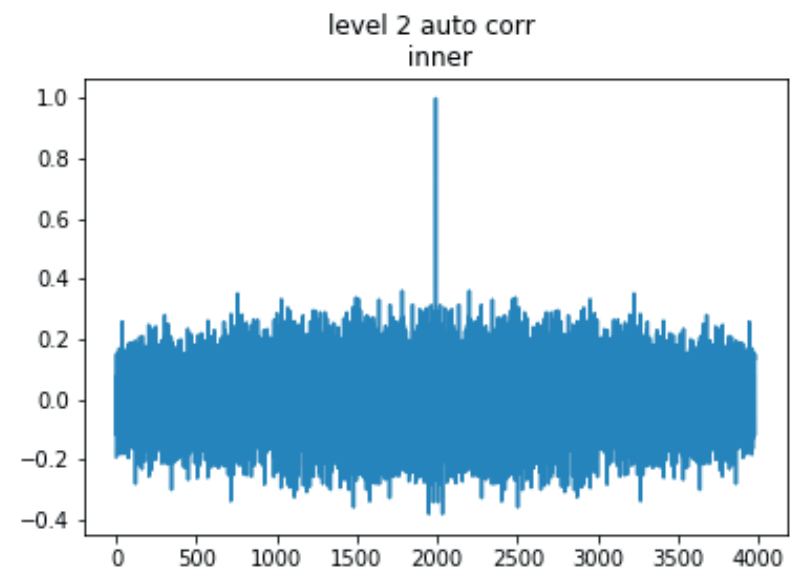

Fig. 23 Second order autocorrelation function of cross-correlation of bands $\mathrm{m}_{1}$ and $\mathrm{m}_{2}$

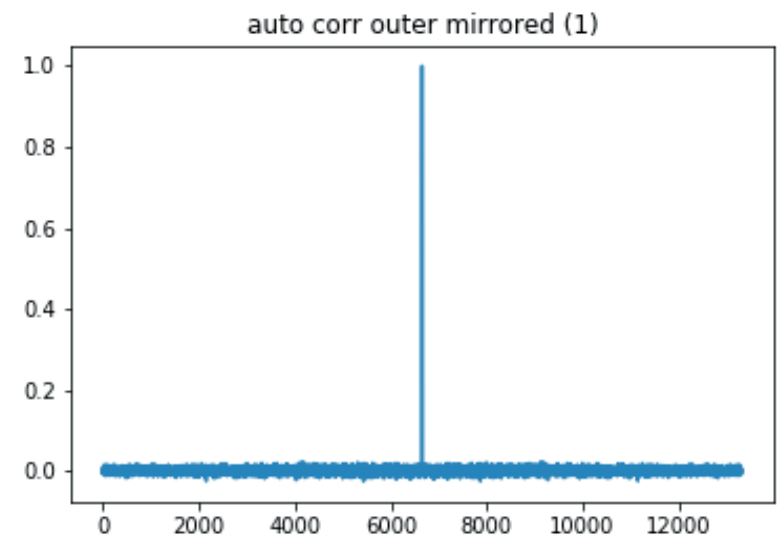

Fig. 24 The autocorrelation function of the band $n_{1}$

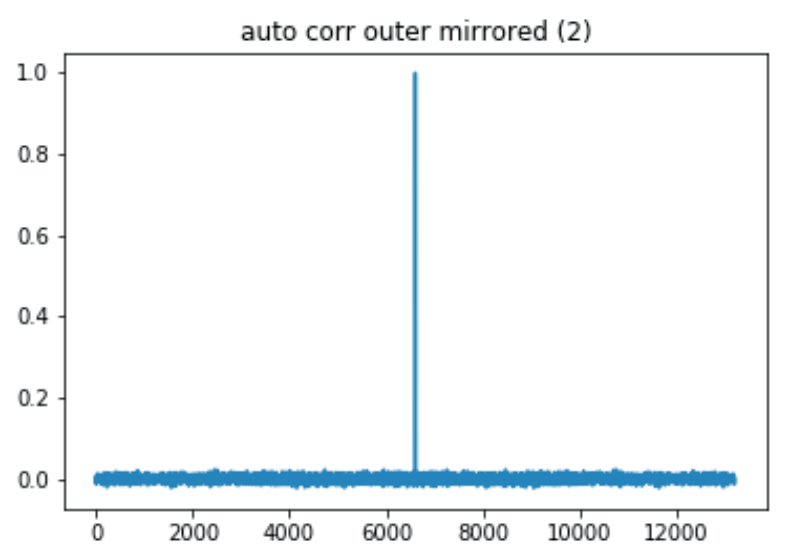

Fig. 25 The autocorrelation function of the band $n_{2}$

"informationlessness" of the theoretical (pure) white noise process represented by the autocorrelation function as well how can we arrive to a periodicity of a hypothetical generative system. Similarly from the increasing characteristic and more individual shapes of the autocorrelation function-realizations with the auto-correlational patterns (mirroring an "intelligent behavior") with 


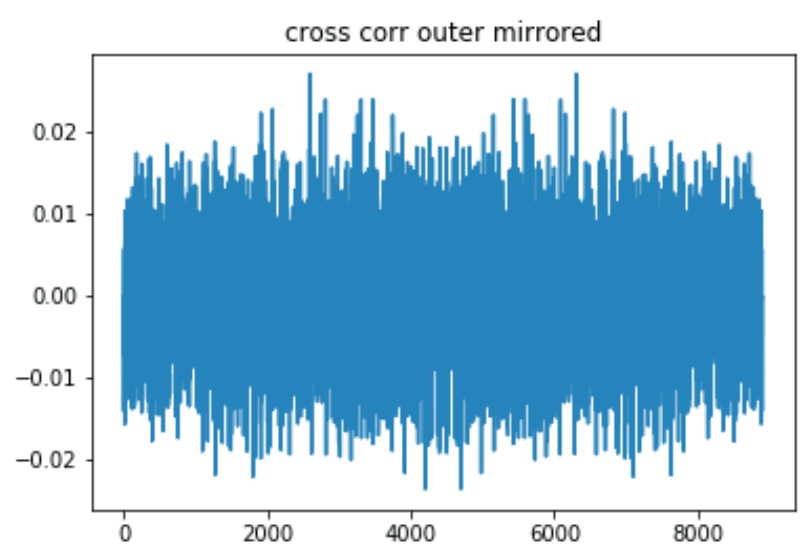

Fig. 26 The cross-correlation of bands $n_{1}$ and $n_{2}$

vanishing stationarity and harmonic periodical-like functions. These observations are deepening the assumption that the undetached observer and the observer's data of the empirical white noise processes consists of a unified system where (as Pauli discussed) a hidden generative controlling behavior with a systole-diastole complementarity (using Goethe's interpretation of Nature and Psyche). It is shown by the phenomena that to begin from the generative process's emanation, diastole series can arrive to an almost perfect "informationless" white noise process. From an inverted point of view the above perfect white noise process can change to a strongly harmonic and regular autocorrelation pattern. Finally, behind this we can live with the hypotheses assuming another "existence" containing number theoretical algorithms. We

\section{References}

Ádám, A., Jánossy, L., Varga, P. (1955) "Coincidences between photons contained in coherent light rays", Acta Physiologica Hungarica, 4, pp. 301-305. (in Russian)

Baranyi, P., Várlaki, P. (2015) "Introducing the Concept of Infotation", In: 2015 6th IEEE International Conference on Cognitive Infocommunications (CogInfoCom), Győr, Hungary, pp. 647-654. https://doi.org/10.1109/CogInfoCom.2015.7390663

Bhargava, A. K. (2013) "Fuzzy Set Theory Fuzzy Logic and their Applications", S. Chand Publishing, New Delhi, India.

Caves, C. M., Fuchs, C. A. Schack, R. (2002) "Unknown quantum states: The quantum de Finetti representation", Journal of Mathematical Physics, 43(9), pp. 4537-4559.

https://doi.org/10.1063/1.1494475

Crełu, M. (2012) "An Optimized Implementation of MWC Pseudorandom Number Generator Analyzed With the Nist Statistical Test Suite for Randomness", Buletinul Institutului Politehnic Din IAŞI, Publicat de Universitatea Tehnică ,Gheorghe Asachi” din Iaşi Tomul LVIII (LXII), Fasc. 2, SecŃia Automatica şi Calculatoare.

De Finetti, B. (1974) "Theory of Probability", Wiley, New York, USA. DeLillo, D. (1985) "White Noise", Viking Press, New York, USA.

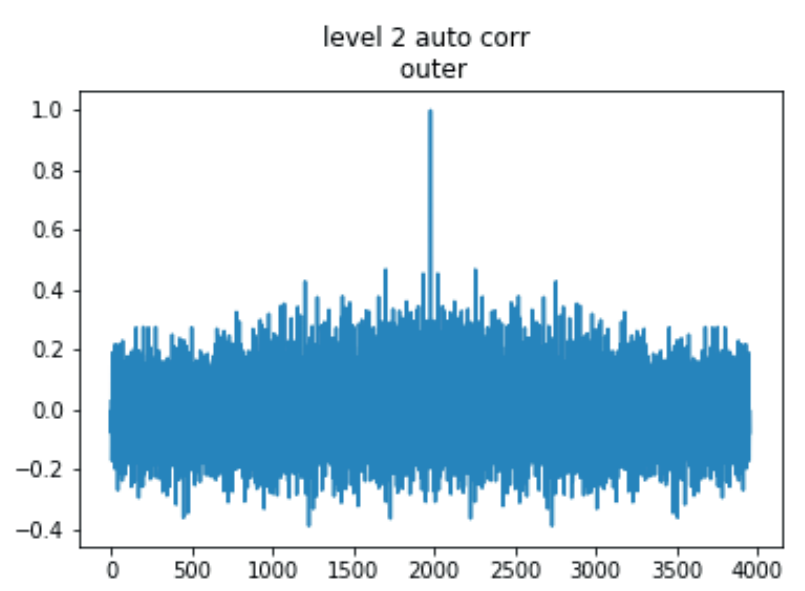

Fig. 27 Second order autocorrelation function of the cross-correlation of bands $\mathrm{n}_{1}$ and $\mathrm{n}_{2}$

have performed additional experiments with further three type of random number generators.

\section{Conclusion}

We may conclude that the introduced fuzzy-random complementary approach can identify the inner hidden correlational pattern of the empirical white noise process if the process has a real hidden structure of this kind. It was shown that these hypothetical hidden correlational patterns inside the process are identifiable in the case of empirical white noise processes generated by deterministic numerical algorithms. We hope that this method can also be applied for real physical empirical white noise processes.

Fullér, R., Harmati, I., Várlaki, P., Rudas, I. (2012) "On Weighted Possibilistic Informational Coefficient of Correlation", International Journal of Mathematical Models and Methods in Applied Sciences, 6(4), pp. 592-599.

Fullér, R., Mezei, J., Várlaki, P. (2011) "An improved index of interactivity for fuzzy numbers", Fuzzy Sets and Systems, 165(1), pp. 50-60. https://doi.org/10.1016/j.fss.2010.06.001

Jung, C. G. (1976) "Psychological Types", Princeton Univ. Press, Princeton, USA.

Keynes, J. M. (1921) "Treaties on Probability", Macmillan \& Co., London, UK.

Kolmogorov, A. N. (1956) "Foundations of the Theory of Probability", Chelsea Publishing Company, New York, USA.

Mathworks (2019) "Random-number-generation", [online] Available at: https://www.mathworks.com/help/matlab/examples/controlling-random-number-generation.html [Accessed: 02 September 2019]

Meier, C. (ed.) (2002) "Atom and Archetype: The Pauli/Jung Letters, 1932-1958", Routledge, London, UK.

Michaletzky, G., Bokor, J., Várlaki, P. (1998) "Representability of Stochastic Systems", Akadémiai Kiadó, Budapest, Hungary. 
von Mises, R. (1964) "Mathematical Theory of Probability and Statistics", Academic Press, New York, USA.

Nietzsche, F., Middleton, C. (ed.) (1996) "Selected letters of Friedrich Nietzsche", Hackett Publishing, Indianapolis, USA.

Papp, Z., Várlaki, P., Nádai, L. (1998) "Wave-Packet Model and Mach-Zehnder-Type Interferometers", In: Hunter, G., Jeffers, S., Vigier, J. P. (eds.) Causility and Locality in Modern Physics. Fundamental Theories of Physics (An International Book Series on The Fundamental Theories of Physics: Their Clarification, Development and Application), Vol. 97., Springer, Dordrecht, The Netherlands, pp. 373-381.

https://doi.org/10.1007/978-94-017-0990-3_44

Pauli, W. (1954) " Wahrscheinlichkeit und Physik" (Probability and Physics), Dialectica, 8(2), pp. 112-124. (in German) https://doi.org/10.1111/j.1746-8361.1954.tb01125.x

Pauli, W. (1994) "Writings on Physics and Philosophy", Springer-Verlag, Berlin, Heidelberg, Germany. https://doi.org/10.1007/978-3-662-02994-7
Popper, K. (1985)"Conjectures and Refutations", Routeledge and Kegan, London, UK.

Savage, L. J. (1954) "The Foundations of Statistics", 2nd ed., Dover Publications, Inc., New York, USA.

Szent-Györgyi, A. (1972) "Dionysians and Apollonians", Science, 176(4038), p. 966.

https://doi.org/10.1126/science.176.4038.966

The Yearbooks of the Hungarian Academy of Sciences, Vol. 16, 18771882, pp. 57-68.

Wikipedia (2019a) "White noise", [online] Available at: https://en.wikipedia.org/wiki/White_noise [Accessed: 02 September 2019]

Wikipedia (2019b) "Bayesian probability", [online] Available at: https:// en.wikipedia.org/wiki/Bayesian_probability [Accessed: 02 September 2019] 